\title{
VMS Mean and Minimum Utilization VM Selection Algorithm for Cloud Datacenter
}

\author{
Sreenivasa B.L, SSathyanarayana
}

\begin{abstract}
Cloud datacenter provides the various resources on-demand for the end consumers on pay-per-use through internet connection. The request for computing resources has increased which leads to the growth of power consumption inside datacenters. The major challenge is how to balance the profits by decreasing the consumption of energy and violation of SLA. VM Consolidation techniques helps VMs to migrate between the host machines in reducing energy consumption, but again which VM to select for migration between the host machine is again the challenging task. In this paper VMs Mean and Minimum Utilization VM Selection Algorithm is proposed.To select the suitable VM's in host machine for the migration, VMs Mean method is used which finds the lowest CPU utilizing VM. To test this algorithm CloudSim Toolkit is been used. The parameter such as EC, SLAV, PDM, ESV, VM migration and SLATAH is used as a metric to evaluate the Algorithm efficiency.
\end{abstract}

Keywords : Energy Consumption (EC), SLA Violation (SLAV), performance degradation due to migration (PDM), Energy SLA Violation (ESV), VM migration and SLA Violation Time per active host (SLATAH), Minimum Utilization (MU), Virtual machine (VM).

\section{INTRODUCTION}

Inpay-per-use model the consumers can purchase the infrastructure, platform and software (applications) as services. The datacenter uses the virtualization technologies to dynamically provision the computing resources. In virtualization, every consumer's, one virtual machine is assigned which executes on host machine present in datacenter. In-efficient utilization of host machine resource leads to wastage of power consumed by host machine. Toenhance the resource consumption and decrease the consumptionof energy in cloud datacenter the virtual machine consolidation is apowerful technique.

Virtual machine consolidation is divided into four separate sub-problems.

Host Overload Detection: determines the host machine, where the virtual machines executing number is more than the host machine capacity. In such cases the few VMs needed to be migrated between the host machines to avoid SLA violation.

Host Underload Detection: determines the host machine,

Revised Manuscript Received on January 30, 2020.

* Correspondence Author

Sreenivasa B.L, Research Scholar Bharathiar university, Coimbatore. $\mathrm{He}$ also works as an Asst Professor at AIMIT, St Aloysius College. Email: srinivasbl.vas@gmail.com

Dr. S Sathyanarayana, First Grade Womens College Mysore, Karnata ka India. Email: ssn_mys@yahoo.com when the lesser number of VMs are executing in the host machine. In such cases the all the Virtual Machines are migrated to new host machine, and current host machine will be shutdown to low power mode.

VM Placement: determines a suitable host machine, to allocate the migrating Virtual Machines from the underloaded host machines or the overloaded host machines.

VM Selection: determines a suitable Virtual Machines which needto be migrated from the overloaded host machine.

In this paper we proposed VMs Mean and Minimum Utilization algorithm, mainly focus on the VM Selectionsub-problem. The migrating VMs from each host machine is collected. The Mean value for these migrating VMs is calculated. Based on the mean value the lowest CPU utilization VM is selected for the migration. By using these VMs the VMP algorithm allocates the suitable host machine for the execution. The main objective is to select the suitable VMs for the migration and also maximize the host machine utilization and efficient energy consumption.

The remaining paper is planned as: Section II discuss the related work to this research. Section III discussion on working of VMs Mean and Minimum Utilization VM Selection algorithm. Section IV explains the simulation setup, metrics for measuring the performance, results and analysis. Section V concludes this research.

\section{RELATED WORK}

During migration process the VMs are unavailable for certain amount of time but it consumes the CPU resources and network from both source and destination host machine.This migration process will have an impact on the other Virtual Machines performance running in the host machine due to increased resource requirements. Therefore a specific VM selection algorithm is needed to select the suitable VMs to migrate between the host machines. In this paper the main work is on VM Selection algorithm, and few works related to it is reviewed in this section.

SuhibBaniMelhem et al [1] proposed Minimum VM Migrated Count and Minimum migration time Minimum VM Migrated Count VM Selection algorithm to avoid regular violation of SLA on the similar Virtual Machine in host machine by selecting the Virtual Machine to migrate from the overloaded host machine based on VM migrated count. The algorithm proves that it reduces the VMs migrated number count and degree of load balancing of VMs migrated count. 
Hanan et al [2] proposed priority-aware virtual machine selection algorithm. The small priority tasks are selected first for the migration. The Hanan et al [2] modified the Minimum Migration Time VM Selection algorithm using the priority concept. The algorithm proves that priority concept has an impact on decliningpower consumption and maximizing SLA violation.

VershaLodhi et al [3] proposed Enhanced Minimum Utilization VM Selection algorithm. The algorithm improve response time and minimize virtual machine migration number. It also increased the utilization of CPU resources in Cloud.

SonalNamdev et al [4] proposed improved minimum migration time VM Selection algorithm. The algorithm minimizes the overall execution time for every host machine. The algorithm proves that it takes less execution time, minimize virtual machine migration number and power consumption.

Zhou et al [5] proposed Adaptive three-threshold energy-aware algorithm (ATEA) Virtual Machine placement algorithm, main goal is reducing consumption of power and violation of SLA. The datacenter host are divided into four categories: host machines with tiny load, host machines with medium load, host machines with modest load, and host machines with dense load. ATEA migrates Virtual Machines on dense loaded or tiny-loaded host machinesto lightly loaded host machines, while the Virtual Machines on medium loaded and modest loaded host machines remain unchanged. Two kinds of adaptive three-threshold algorithm and three kinds of VM selection policies such as Minimum Memory Size algorithm (MMS), Lowest CPU Utilization algorithm (LCU), Minimum Product of Both CPU Utilization and Memory Size algorithm (MPCM) are proposed.The algorithms prove that dynamic thresholds are more power efficient than fixed threshold.

Mohammad AlaulHaqueMonil et al [6] proposed incorporating migration control in VM selection strategies to enhance performance. In this algorithm the usage of CPU has been considered to find a Virtual Machine to be study or not. The VM Selection mechanisms are modified and equations are also altered to implement migration control in the policy. The Minimum Migration Time with Migration Control algorithm, Maximum Correlation with Migration Control algorithm, Random Selection with Migration Control algorithm of VM Selection are proposed. The algorithm proves that performance is enhanced than other proposed heuristics approaches.

\section{VMS MEAN AND MINIMUM UTILIZATION ALGORITHM}

In this subsection, a VMs Mean and Minimum Utilization Algorithm is designed as a solution for the Virtual Machine Selection problem. The host machine provisioned by the datacenter gets overloaded when the capacity of host machine executing the virtual machine number exceeds, the host machine gets under-loadedwhen virtual machine number are lesser in execution. In that case the performance degrades giving rise to SLA violation. The solution to this problem is selecting the suitable Virtual Machine for migration from one host machine to another host machine.
To design an algorithm the factor considered here is to select the suitable virtual machine with lowest CPU utilization. First for each host machine the Virtual machines ready for migration is collected and stored in the vmList. These migration ready virtual machines are sorted in the order of decreasing MIPS. Mean value for the virtual machines in the vmlist is calculated using equation (1). Virtual machine with lower CPU utilization is selected for the migration.

After sorting the migrating vmList in the order of decreasing MIPS. Mean value is calculated using equation (1). The larger the mean the larger the CPU utilization.

$$
\text { meanVMList }=\left(\sum_{k=0}^{n} V M^{k}\right) / \text { VMListSize }
$$

TABLE 1: SYMBOLIZATION TABLE

\begin{tabular}{|l|l|}
\hline EXPRESSION & DESCRIPTION \\
\hline vmList & $\begin{array}{l}\text { LIST OF } \\
\text { MIGRATING } \\
\text { VM'S }\end{array}$ \\
\hline vmToMigrate & $\begin{array}{l}\text { VM suitable for the } \\
\text { migration }\end{array}$ \\
\hline mean & $\begin{array}{l}\text { Mean of VMsCPU } \\
\text { utilization in vmList }\end{array}$ \\
\hline totalCPUUtilization & $\begin{array}{l}\text { Total CPU } \\
\text { utilization of VMSs }\end{array}$ \\
\hline getTotalUtilizationOfCPU(Cloud & $\begin{array}{l}\text { VM total CPU } \\
\text { utilization }\end{array}$ \\
\hline Sim.Clock()) & VM total MIPS \\
\hline getMIPS() & \multicolumn{2}{|c|}{ C } \\
\hline
\end{tabular}

\section{Algorithm 1: VMs Mean and Minimum Utilization Algorithm}

Input: overloaded Host

Output: vm selected for migration

1 Get the list of VMs to migrate from the host;

2 ifvmList is empty then

3 I return null

$4 \quad$ vmToMigrate $\leftarrow$ null;

5 totalCPUUtilization $\leftarrow 0$;

6 foreach VM in vmListdo

3 totalCPUUtilization $=$ totalCPUUtilization + VMCPUUtilization

4calculate mean for VMList

5 foreach VM in vmListdo

6 if vmisInMigration then

7 getTotalUtilizationOfCPU(CloudSim.Clock()) /

vm.getMIPS() value of vm and allocate it to variable Metric

8 if value of Metric is lesser the mean then

9 vmToMigrate $\leftarrow$ vm;

10 return vmToMigrate 


\section{TESTING ENVIRONMENT}

\section{A. Simulation setup}

The performance of VMs Mean and Minimum Utilization algorithm compared against the three other VM Selection algorithms using CloudSim 3.0.3 toolkit. Simulation uses 800 heterogeneous host machine, half of which are HP ProLiant G4 and remaining half are HP ProLiant G5 servers. Energy consumption for G4 and G5 servers are listed in Table 2. Server's configuration are listed in Table 3. VM's configuration are listed in Table 4. For this simulation the data set we have used real world work load data provided from CoMon Project, a monitoring infrastructure for PlanetLab is listed in Table 5.

\section{B. Metrics for Measuring Performance}

To study the progress of the algorithm the following metrics are used.

1. Energy consumption - sum of the host machines energy consumption in the datacenter.

2. SLAV - are the violation in the Service Level Agreement's. If the requirements of the cloud consumers are not met, then SLA violation we get.

3. SLATAH - summing up all the violation for each host machine when the assigned MIPS is less than the demanded MIPS.

4. PDM - average performance degradation caused in each host due to migrations.

5. VM migration numbers - VM's selected for migration from overloaded or under-loaded hosts.

6. ESV - combination of power consumption and violation of SLA.

Table 2Energy consumption by the selected servers at different load levels in Watts

\begin{tabular}{|l|l|l|l|l|l|l|}
\hline \multirow{2}{*}{$\begin{array}{l}\text { Machine } \\
\text { Type }\end{array}$} & \multicolumn{6}{|c|}{ Power Consumption Based on CPU } \\
\cline { 2 - 7 } & $0 \%$ & $20 \%$ & $40 \%$ & $60 \%$ & $80 \%$ & $100 \%$ \\
\hline $\begin{array}{l}\text { HP G4 } \\
\text { (Watt) }\end{array}$ & 86 & 92.6 & 99 & 106 & 112 & 117 \\
\hline $\begin{array}{l}\text { HP G5 } \\
\text { (Watt) }\end{array}$ & 93.7 & 101 & 110 & 121 & 129 & 135 \\
\hline
\end{tabular}

Table 3 Server'sConfiguration

\begin{tabular}{|l|l|}
\hline $\begin{array}{l}\text { Machine } \\
\text { Type }\end{array}$ & Description \\
\hline HP G4 & $\begin{array}{l}\text { 1860MIIP, 4 GB Ram, 1 GB Storage, 1 GB } \\
\text { network bandwidth. }\end{array}$ \\
\hline HP G5 & $\begin{array}{l}\text { 2660MIIPS, 4 GB Ram, 1 GB Storage, 1 GB } \\
\text { network bandwidth. }\end{array}$ \\
\hline
\end{tabular}

Table 4 Virtual Machines Configuration

\begin{tabular}{|l|l|}
\hline VM types & Description \\
\hline $\begin{array}{l}\text { High-CPU } \\
\text { Medium } \\
\text { Instance }\end{array}$ & $\begin{array}{l}\text { Storage, 100 Mbit's network bandwidth. } \\
\text { Extra Large }\end{array}$ \\
Instance & $\begin{array}{l}2000 \mathrm{MIPS}, 1740 \mathrm{MB} \text { Ram, 2.5 GB } \\
\text { Storage, 100 Mbit's network bandwidth. }\end{array}$ \\
\hline $\begin{array}{l}\text { Small } \\
\text { Instance }\end{array}$ & $\begin{array}{l}1000 \mathrm{M} \text { MS, 1740 MB Ram, 2.5 GB } \\
\text { Storage, 100 Mbit's network bandwidth. }\end{array}$ \\
\hline Micro & $500 \mathrm{MPS}, 613 \mathrm{MB}$ Ram, 2.5 GB Storage, \\
Instance & $100 \mathrm{Mbit} / \mathrm{s}$ network bandwidth. \\
\hline
\end{tabular}

Table 5. Selected trace-based workloads.

\begin{tabular}{|l|l|l|l|}
\hline Workload & $\begin{array}{l}\text { No. of } \\
\text { VMs }\end{array}$ & Workload & $\begin{array}{l}\text { No. of } \\
\text { VMs }\end{array}$ \\
\hline 20110303 & 1052 & 20110403 & 1463 \\
\hline 20110306 & 898 & 20110409 & 1358 \\
\hline 20110309 & 1061 & 20110411 & 1233 \\
\hline 20110322 & 1516 & 20110412 & 1054 \\
\hline 20110325 & 1078 & 20110420 & 1033 \\
\hline
\end{tabular}

\section{Simulation result and Analysis}

CloudSim toolkit is used for the simulation. The proposed VMs Mean and Minimum Utilization algorithm is compared with other three VM Selectionalgorithms such as Enhanced Minimum Utilization algorithm, Lowest CPU Utilization algorithm, Minimum Utilization algorithmas described in Table 6. For this experiment VMs Mean and FFD algorithm is used as a VM Placement algorithm, Local Regression (LR) as overload detection algorithm and proposed VMs Mean and Minimum Utilization as VM Selection algorithm. The simulation results shows our proposed algorithm givesenhanced result is selecting least CPU Utilization Virtual machine, by using this Virtual machine the VM Placement algorithm is able to achieve the objective of maximizing the CPU Utilization with efficient energy consumption.

Discussion on Analysis of proposed algorithm along with other three VM Selection algorithm are given below.

1. Energy consumption - the proposed algorithm consumes energy less compared to remaining VM Selection algorithm Figure 1 shows the result of the consumption of energy.

2. SLA violation -the proposed algorithm shows low violation of SLA, ensures desired QoS. Figure 2 shows the result of the SLA violation.

3. SLATAH - The proposed algorithm have minimum SLA violation time per active host. Figure 3 shows the result of SLATAH.

4. PDM - the proposed algorithm shows less performance degradation due to migration. Figure 4 shows the result of PDM.

5. VM migration number - the EMU algorithm have less number of migrating VM's. Figure 5 shows the result of VM migration number.

6. ESV - the proposed algorithm have less combined energy and SLA violation. Figure 6 show the result.

From the above analysis the proposed algorithm gives good result than the other three VM Selection algorithm with respect to all the metrics used for measuring the performance. It proves that the proposed algorithm gives enhanced result is selecting least CPU Utilization Virtual machine, by using this Virtual machine the VM Placement algorithm is able to achieve the objective of maximizing the CPU Utilization with efficient energy consumption. 
VMS Mean and Minimum Utilization VM Selection Algorithm for Cloud Datacenter

Table 6. Summary of all the VM placement Algorithms

\begin{tabular}{|l|l|l|l|l|}
\hline & MU & LCU & EMU & $\begin{array}{l}\text { VMs } \\
\text { Mean } \\
\text { and MU }\end{array}$ \\
\hline $\begin{array}{l}\text { Energy } \\
\text { Consumption } \\
(\mathrm{kWh})\end{array}$ & 110.69 & 111.14 & 111.27 & 110.26 \\
\hline $\begin{array}{l}\text { VM } \\
\text { Migration }\end{array}$ & 12915 & 15331 & 7003 & 9699 \\
\hline SLATAH & $9.41 \%$ & $8.38 \%$ & $7.94 \%$ & $7.18 \%$ \\
\hline PDM & $0.02 \%$ & $0.02 \%$ & $0.02 \%$ & $0.01 \%$ \\
\hline $\begin{array}{l}\text { SLAV } \\
\left(10^{-2}\right)\end{array}$ & 0.00141 & 0.00140 & $0.00149 \%$ & 0.00100 \\
\hline $\begin{array}{l}\text { ESV } \\
\left(10^{-2}\right)\end{array}$ & 0.15 & 0.15 & 0.16 & 0.11 \\
\hline $\begin{array}{l}\text { Average } \\
\text { SLA } \\
\text { Violation }\end{array}$ & $10.32 \%$ & $10.22 \%$ & $9.42 \%$ & $9.45 \%$ \\
\hline $\begin{array}{l}\text { Number of } \\
\text { Host } \\
\text { Shutdowns }\end{array}$ & 814 & 818 & 815 & 805 \\
\hline
\end{tabular}

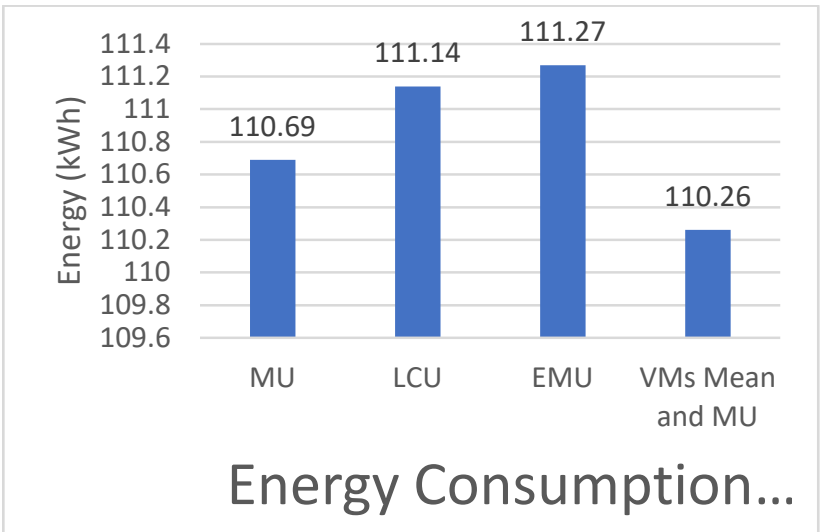

Figure 1 Energy Consumption Chart

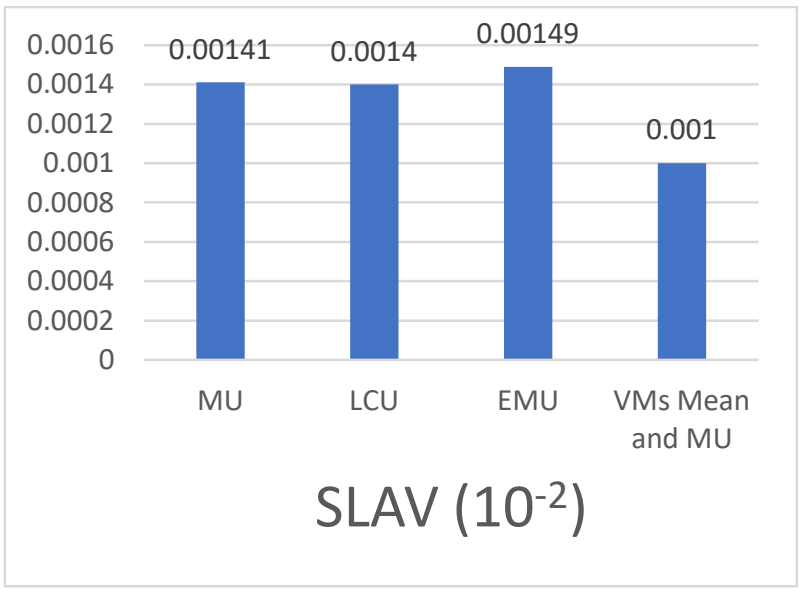

Figure 2 SLA Violation Chart

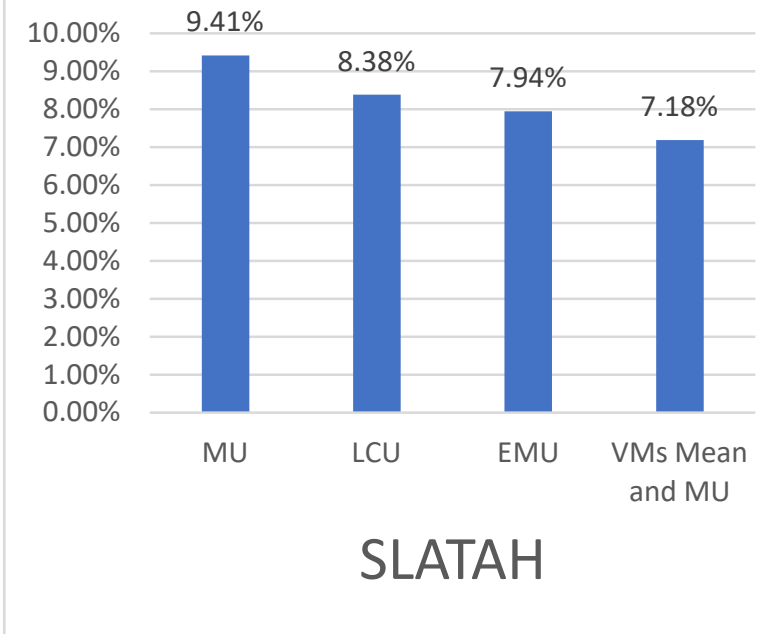

Figure 3 SLATAH Chart

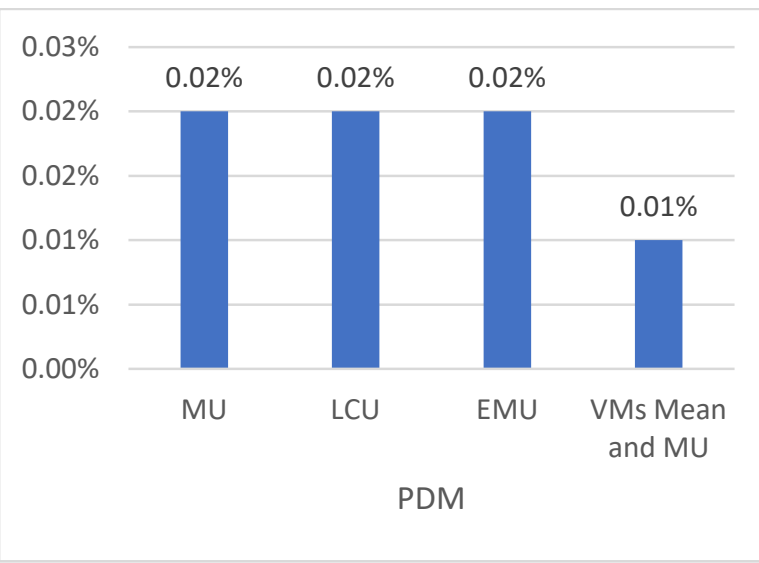

Figure 4 PDM Chart

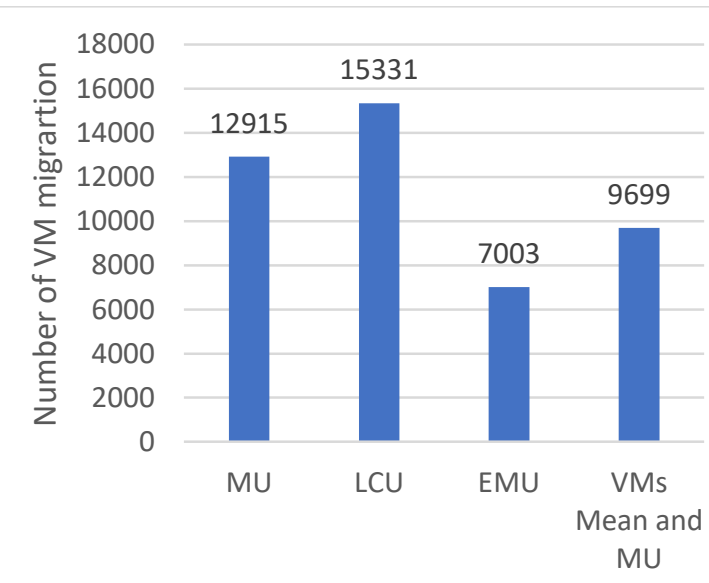

VM Migration

Figure 5 VM Migration Chart 


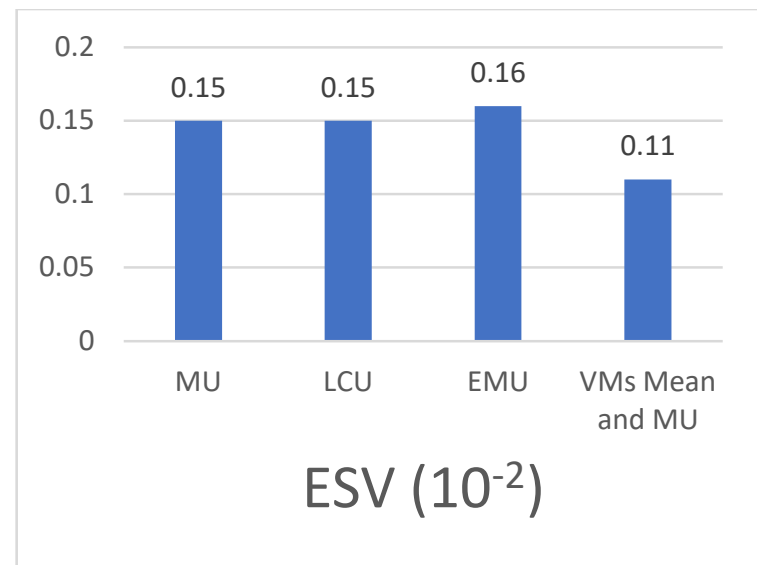

Figure 6 ESV Chart

\section{CONCLUSION}

The proposed VMs Mean and Minimum Utilization algorithm computes the mean value for the migration VMs List for each host machine. CPU utilization of each VM is compared with the mean value. Least CPU utilization of VMs is selected for the migration. It proves that the proposed algorithm gives enhanced result is selecting least CPU Utilization Virtual machine, by using this Virtual machine the VM Placement algorithm is able to achieve the objective of maximizing the CPU Utilization with efficient energy consumption.

\section{REFERENCES}

1. SuhibBaniMelhem, Anjali Agarwal, NshithGoel, MarziaZaman. "' Minimizing Biased VM Selection in Live VM Migration", 978-1-5386-1115-9/17/\$31.00@2017 IEEE.

2. Suhib Bani Melhem, Anjali Agarwal, Nshith Goel, Marzia Zaman. " Minimizing Biased VM Selection in Live VM Migration”, 978-1-5386 -1115-9/17/\$31.00@2017 IEEE.

3. Hanan A. Nadeem, Mai A. Fadel and Hanan Elazhary, "Priority-Awar e Virtual Machine Selection Algorithm in Dynamic Consolidation", (IJ ACSA) International Journal of Advanced Computer Science and Appl ications, Vol. 9, No. 11, 2018.

4. Versha Lodhi, Prof. Sarvesh Rai, Ganesh Kumar Vishwakarma, "Enha nced Minimum Utilization VM Selection Mechanism for Clouds", (IJ CSIT) International Journal of Computer Science and Information Tec hnologies, Vol. 6 (3) , 2015, 2975-2977.

5. Sonal Namdev, Prof. Neelam Sain, Prof. Anjul K S Rai, “ Improved M inimum Migration Time VM Selection Policy for Cloud Data Center”, International Journal of Application or Innovation in Engineering \& $\mathrm{M}$ anagement (IJAIEM), www.ijaiem.org Email: editor@ijaiem.org, Volu me 4, Issue 4, April 2015 ISSN 2319 - 4847

6. Zhou Zhou, Zhigang $\mathrm{Hu}$, and Keqin Li, "Virtual Machine Placem ent Algorithm for Both Energy-Awareness and SLA Violation Re duction in Cloud Data Centers", Hindawi Publishing Corporation Scientific Programming Volume 2016, Article ID 5612039, 11 pa ges http://dx.doi.org/10.1155/2016/5612039

7. Mohammad Alaul Haque, Monil, Romasa Qasim, Rashedur M Rahma $\mathrm{n}$, "Incorporating Migration Control in VM Selection Strategies to Enh ance Performance", International Journal of Information Web Applicat ions Volume 6 Number 4 December 2014.

8. Anton Beloglazov a, Jemal Abawajyb, Rajkumar Buyyaa, "Energ $\mathrm{y}$-aware resource allocation heuristics for efficient management of data centers for Cloud computing", 0167-739X/\$ - see front $\mathrm{m}$ atter (C) 2011 Elsevier B.V. All rights reserved. doi:10.1016/j.futu re. 2011.04.017

9. L. Shi, J. Furlong, and R. Wang. "Empirical evaluation of vector bin $\mathrm{p}$ acking algorithms for energy e_cient data centers", In IEEE Symposiu $\mathrm{m}$ on Computers and Communications, pages 9\{15, 2013.

10. Jay H. Sheth, Prof. Krunal N. Vaghela, "Technical Review on Live Virtual Machine Migration Techniques for Eucalyptus Cloud", Jay H. Sheth Int. Journal of Engineering Research and Applic ations, ISSN : 2248-9622, Vol. 5, Issue 3, ( Part -2) March 2015, pp.50-52.
11. Server Virtualization: A step toward Cost Efficiency and Business Agi lity, Avanade perspective, 2009.

12. Dabiah Ahmed

Alboaneen1, Bernardi Pranggono and Huaglory Tianfield,“ Energy-awa re Virtual Machine Consolidation for Cloud Data Centers2014 IEEE/ACM 7th International Conference on Utility and Cloud Computing.

13. Nicolo Maria Calcavecchia, Ofer Biran, Erez Hadad, and Yosef Moatti VM placement strategies for cloud scenarios. In IEEE

5 th International Conference on Cloud Computing (CLOUD), pages $852\{859,2012$.

\section{AUTHORS PROFILE}

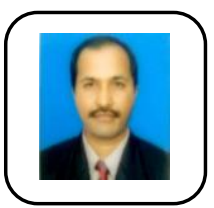

Sreenivasa B.L, Pursued his master degree Master of Science in Computer Science from Kuvempu University. Currently he is a research scholar in Bharathiar University Coimbatore and also as an Asst Professor in AIMIT, St Aloysius college, Mangalore. His interest of research is Cloud Computing.

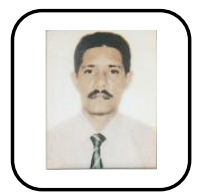

Dr S Sathyanarayana, Completed his BTech, Msc and Phd. His area of interest is Decision Support system and Cloud Computing, Relational Database Management Ssystems. 